\title{
Quantitative application of pulse phase thermography to determine material parameters
}

\author{
by B. Stotter*, K.H. Gresslehner*, G. Mayr*, G. Hendorfer* and J. Sekelja**
}

* University of Applied Sciences Upper Austria, Research and Development Ltd., Stelzhamerstraße 23, 4600 Wels, Austria, bernhard.stotter@fh-wels.at

${ }^{\star *}$ FACC AG, Fischerstraße 9, 4910 Ried im Innkreis, Austria, j.sekelja@facc.com

\begin{abstract}
This work presents a method to determine material parameters of pulse thermography data transformed form the time domain into the frequency domain. The procedure for determining the material parameters is based on a 1D solid model. At first a physically correct and piecewise continuous trial function is fitted to the measured temperature curve in the time domain. At next the trial function is transformed in the frequency domain by Integral Fourier transformation. In the frequency domain the Green's function method is used to solve the heat conduction equation analytically. To determine material parameters of the inspected specimen, the phase curve of the analytical solution of the heat conduction equation is fitted to the phase curve of the transformed trial functions. Using this method, the parameters $\omega_{0}, B_{1}$ and $H$ can be determined which correspond to the material parameters $L, \alpha$ and $k$ as well as the heat transfer coefficients $h_{1}$ and $h_{2}$.
\end{abstract}

\section{Introduction}

The development of new manufacturing techniques and the use of new high-tech materials enable the industrial production of evermore complex structures. Defects, such as impact damages, delamination or cracks, have to be found in quality controls after manufacturing. Due to this development, the requirements for non-destructive inspection methods are increasing rapidly. In the recent past active thermography has established as a non-destructive inspection method in many industries. Previous studies have shown that active thermography, i.e. pulse phase thermography (PPT), is an effective method for detecting flaws in Carbon Fibre Reinforced Plastics (CFRP). One advantage of PPT is that, due to the pulse excitation, a wide frequency spectrum is tested simultaneously [1,9]. Currently, PPT is used mainly for qualitative analyses. In order to enable quantitative assessments of the inspected specimen, a method used to determine material parameters is discussed herein this paper.

\section{Parameter Reconstruction in Frequency Domain (PRFD)}

The PRFD procedure is based on the principle of the PPT method evaluated especially for the reflection mode. The inspected specimen is stimulated by a pulse excitation on the front side of the specimen. Thereby, a wide frequency spectrum can be evaluated within one investigation [1,9]. During the measurement of the temperature versus time profile on the front side of the specimen, the temperature data are recorded in discrete time steps depending on the frame rate. To obtain a continuous function of the temperature profile physically correct trial functions are fitted to the recorded data. Later on the three trial functions are combined into one continuous trial function. Using an Integral Fourier transformation (IFT) this continuous temperature curve is transformed into the frequency domain. One advantage of evaluating the recorded temperature data in the frequency domain is the existing analytically solution of the Fourier heat equation. The determination of the material parameters by the PRFD procedure is carried out by fitting the phase curve of the analytical solution to the phase curve of the Fourier transformed trial function. This has the advantage that the excitation energy is cancelled out in the phase slope. The PRFD procedure was developed and evaluated by using simulated data and applied on experimental measurements. The detailed description of the PRFD procedure is given in the following sections.

\subsection{Time domain}

In the time domain the heat conduction equation with the used boundary conditions (Eqs. (2) and (3)) and initial condition (Eq. (4)) for the one dimensional problem reads

$$
\nabla^{2} T(x, t)-\frac{1}{\alpha} \frac{\partial}{\partial t} T(x, t)=-\frac{1}{k} q(x, t) .
$$




$$
\begin{gathered}
\left.\vec{n}(k \nabla T)\right|_{x=0}=q_{0}+h_{1}\left(T_{\infty}-T\right) \\
\left.\vec{n}(k \nabla T)\right|_{x=L}=h_{2}\left(T_{\infty}-T\right) \\
T(x, 0)=T_{0}
\end{gathered}
$$

In Eq.(1) q $\left[\mathrm{Wm}^{-3}\right]$ is the energy generation term, $\alpha\left[\mathrm{m}^{2} \mathrm{~s}^{-1}\right]$ is the thermal diffusivity and $k\left[\mathrm{Wm}^{-1} \mathrm{~K}^{-1}\right]$ is the thermal conductivity in the region of space where Eq.(1) holds.

In Eq.(2) and (3) $q_{o}\left(W / m^{2}\right)$ is the incident heat flux, $h_{1}$ and $h_{2}$ are the heat transfer coefficients at $x=0$ and $x=L$ respectively, $T_{\infty}$ is the ambient temperature, $T_{0}$ the initial temperature and $L$ the sample thickness. $n$ denotes the normal vector at the boundaries.

As the parameter determining procedure of the PRFD procedure is carried out in the frequency domain, the recorded temperature curve has to be transformed into the frequency domain. Due to the nature of the measurement, the temperature curve is recorded in discrete time steps, depending on the frame rate. As a result of this, only discrete Fourier transform (DFT) is suitable to transform the recorded data into the frequency domain directly. When using DFT, the following two parameters must be considered. $[2,3]$

- The measurement duration determines the frequency resolution.

- The sampling frequency determines the maximum frequency.

These parameters can falsify and distort the phase slope in the frequency domain (see figure $5 b$ ). To keep the influence of the falsification as low as possible a long measurement duration with a simultaneous high sampling frequency is required. Due to the long duration and the huge amount of data, this would be very inefficient in practise. The influence of these parameters is elucidated particularly in $[2,3]$.

To avoid the difficulties of DFT, in the PRFD- procedure three physically correct functions are fitted to the recorded data in the respective applicable sectors to obtain one continuous function. Therefore, the simulated temperature profile is divided into three time sections. Figure 1 shows a simulated temperature profile (black dots). The dashed lines mark the divided sections. $t_{1}$ and $t_{2}$ are the so-called switch times which are used to fit the temperature curve with the appropriate part of the trial function.

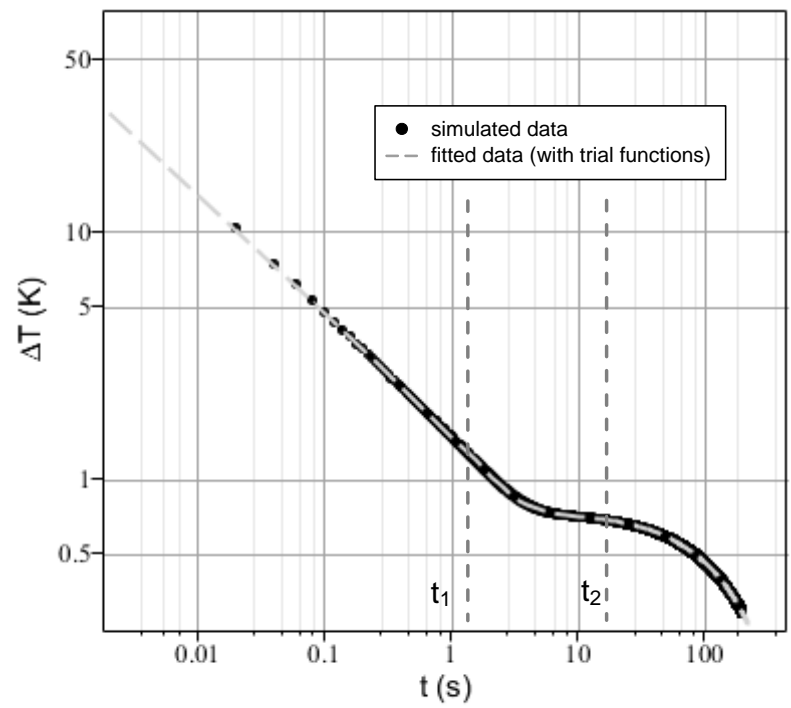

Fig. 1. Simulated Temperature profile and fitted by trial function for a solid of thickness $L=2.23 \mathrm{~mm}$. The switch times (dotted lines) are set at $t_{1}=1.21 \mathrm{~s}$ and $t_{2}=14.57 \mathrm{~s}$ for this fitting procedure.

The examined temperature profiles refer to the used boundary conditions given in Eq.(2) and (3). Shortly after the pulse excitation the, conditions of a semi-infinite body are fulfilled $\left(t<t_{1}\right)$. Therefore in the first section the temperature profile must follow a $t^{-1 / 2}$ law. In the third section $\left(t>t_{2}\right)$ only the convection on the two boundaries is relevant for describing the temperature profile. In this section the temperature profile follows an exponential decay. In the middle section $\left(t_{1}<t<t_{2}\right)$ the temperature profile can be described mathematically as a combination of the exponential- and the $\mathrm{t}^{-1 / 2}$ behavior. The functions for the respective applicable sectors are shown below. Thereby $A_{i j}$ and $B_{i j}$ are the fitting parameters for the specific regions. 


$$
T(t)=\left\{\begin{array}{cc}
\frac{A_{11}}{\sqrt{A_{12} t+1}}+B_{11} e^{-B_{12} t} & 0 \leq t \leq t_{1} \\
A_{21} \frac{A_{22}}{\sqrt{t}}+B_{21} e^{-B_{22} t} & t_{1}<t \leq t_{2} \\
B_{31} e^{-B_{32} t} & t_{2}<t
\end{array}\right.
$$

The exponential decay with the fitting parameters $B_{11}$ and $B_{12}$ in the first section, acts as a damping term in the fitting procedure. The influence of this exponential decay is very low in this section, the values of the parameters are approximately 0.1 for $\mathrm{B}_{11}$ and $>100$ for $\mathrm{B}_{12}$. The scope of application for the respective trial function depends on the material parameters of the inspected specimen. After fitting these functions to the recorded data in the respective scope, the functions are combined into one continuous trial function. Thereby discontinuities at $t_{1}$ and $t_{2}$ may occur. These discontinuities have an influence on the phase profile in the frequency domain. To avoid these discontinuities a linear interpolation between one time step before and one time step after the corresponding switch time is used to smooth the curve (figures $2 a$ and $2 b$ ).

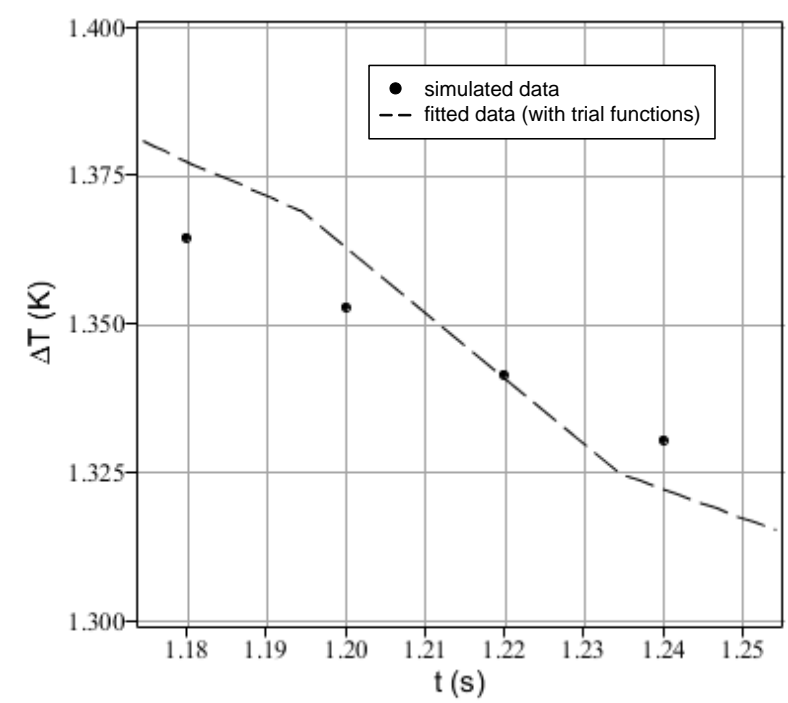

a) Linear interpolation at $t_{1}$

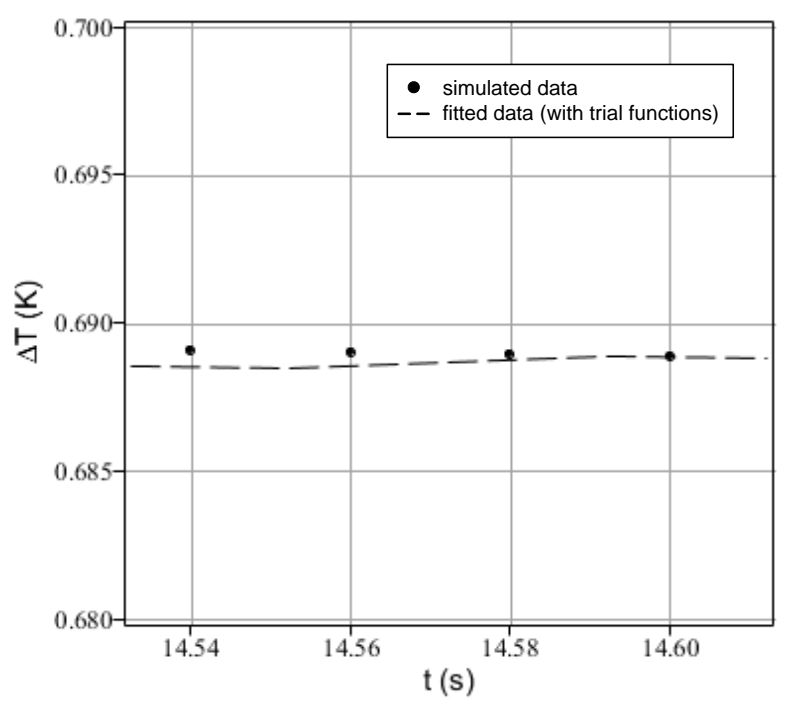

b) Linear interpolation at $t_{2}$

Fig. 2. Linear interpolations at the stitching areas. The linear interpolation is performed in-between one time step before and one time step after the stitching time $t_{i}$.

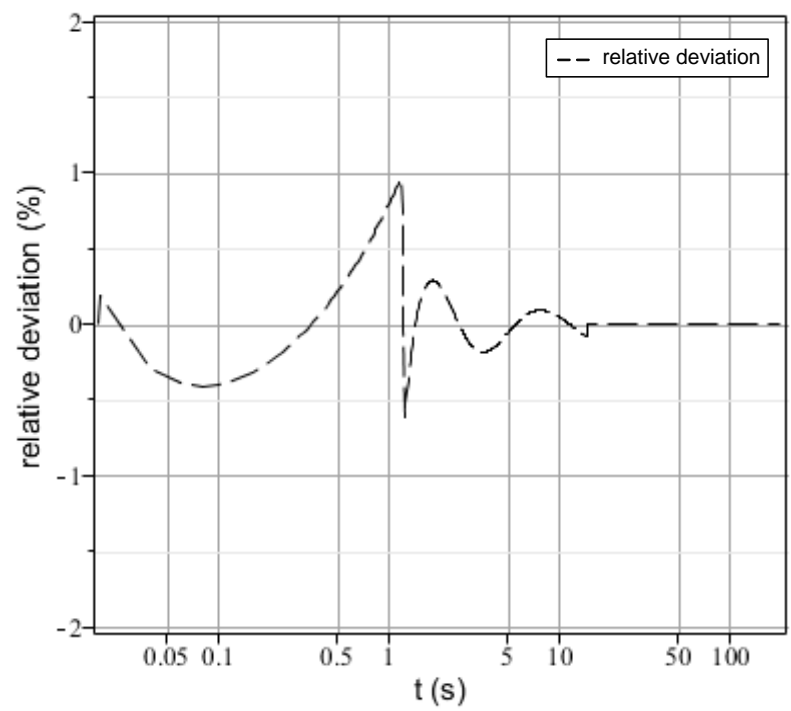

Fig. 3. Relative deviation of the trial function to the recorded temperature data. 
The relative deviation of the fitted trial function from the simulated data is shown in figure 3 . The relative deviation is less than $1 \%$ over the total time range which is an excellent agreement of the trial function with the simulated data. Now the continuous trial function can be transformed into the frequency domain by the IFT without the influences of the DFT.

\subsection{Frequency domain}

Taking the Fourier transform of Eq. (1), performing an integration by parts in the energy generation term and using boundedness conditions $T(x, t)$ at $t \rightarrow \pm \infty$ results in the Fourier heat Equation in the frequency domain. The equation is given by

$$
\nabla^{2} T(x, \omega)-\sigma^{2}(\omega) T(x, \omega)=-\frac{1}{k} Q(x, \omega) .
$$

In this equation $Q(x, \omega)$ is the Fourier transform of the energy generation term $q(x, t)$ and $\sigma\left[\mathrm{m}^{-1}\right]$ has the physical meaning of a dispersive complex wavenumber characterized by

$$
\sigma(\omega)=(1+i) \sqrt{\frac{\omega}{2 \alpha}}
$$

In comparison to the time domain, the heat conduction equation can be solved analytically in the frequency domain, without using series expansions. In the PRFD procedure, the Green's function for a one dimensional case and homogeneous boundary conditions of the third kind in the region $0 \leq x \leq L$ with a spatially impulse thermal-wave source at $x_{0}(\mathrm{Eq} .(8))$ is used to solve the heat conduction equation in the frequency domain (Eq. (6)) $[4,5]$.

$$
G\left(x \mid x_{0} ; \omega\right)=\frac{1}{2 \alpha \sigma}\left[\frac{e^{-\sigma\left|x-x_{0}\right|}+R_{1} e^{-\sigma\left(x+x_{0}\right)}+R_{1} R_{2} e^{-\sigma\left[2 L-\left|x-x_{0}\right|\right]}+R_{2} e^{-\sigma\left[2 L-\left(x+x_{0}\right)\right]}}{1-R_{1} R_{2} e^{-2 \sigma L}}\right]
$$

In Eq. (8) $R_{1}$ and $R_{2}$ are the surface thermal wave power transfer coefficients on the front and on the back side of the specimen respectively. The temperature decay in the frequency domain can be described mathematically by $a$ convolution of the Green's function with the excitation source. For the temperature profile on the front side of the specimen the following equation applies.

$$
\tilde{T}(0, \omega)=\tilde{Q}_{0}^{*}(\omega) \frac{1}{\sigma^{*}} \cdot \frac{\left(1+R_{1}\right)\left(1+R_{2} e^{-2 \sigma^{*}}\right)}{1-R_{1} R_{2} e^{-2 \sigma^{*}}}
$$

For Eq. (9) following requirements shall apply.

$$
\begin{gathered}
\sigma^{*}=L \sigma(\omega) \\
\widetilde{Q}_{0}^{*}(\omega)=Q(\omega) \cdot \frac{L}{k}
\end{gathered}
$$

That implies that the temperature profile depends on six material parameters $\left(k, h_{1}, h_{2}, L, \alpha\right.$ and $\left.Q\right)$. As the PRFD procedure leads to a fitting procedure of the phase slope the number of fitting parameters are reduced. As a consequence of analysing the phase slope, which is calculated by

$$
\tan (\varphi)=\left(\frac{\operatorname{Im}[\tilde{T}(0, \omega)]}{\operatorname{Re}[\tilde{T}(0, \omega)]}\right)
$$

Due to this, the intensity of the excitation energy cancels out. The further aggregation of the material parameter is explained explicitly in [2]. For the fitting procedure of the phase slope only the following three parameters must be taken into account [2].

$$
\begin{aligned}
& \omega_{0}=\frac{\alpha}{L^{2}} \\
& B_{1}=h_{1} \frac{L}{k} \\
& H=\frac{h_{2}}{h_{1}}
\end{aligned}
$$


In the PRFD procedure the fitting procedure is carried out in three steps. At first the high frequency domain is investigated. The high frequency range is defined where $\Omega \gg B_{1}$ holds. $\Omega$ is an auxiliary variable and is given by

$$
\Omega=\sqrt{\frac{\omega}{2 \omega_{0}}} .
$$

In the high frequency range the influence of the parameters $\mathrm{B}_{1}$ and $\mathrm{H}$ are negligible and are set to zero. Consequently the phase slope in the high frequency range is only depending on parameter $\omega_{0}$. Therefore, a fit of the analytical solution in the high frequency range is used to determine $\omega_{0}$. The fit in the high frequency range is shown in figure 4 , whereby the region in-between the two dotted lines represent the high frequency range.

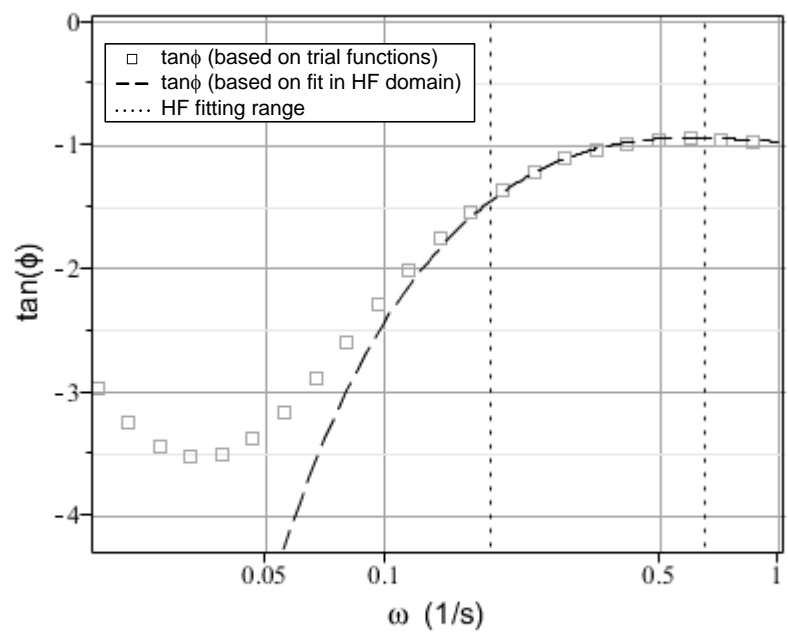

Fig. 4. Fitting procedure of the phase slope in the high frequency range to determine parameter $\omega_{0}$ whereby $B_{1}$ and $H$ are set to be zero.

In the next step a fit in the low frequency range is carried out to determine the remaining parameters $\mathrm{B} 1$ and $\mathrm{H}$. Thereby $\omega_{0}$ is kept constant and the parameter $\mathrm{H}$ is bounded in a range of $0<\mathrm{H} \leq 0.48$. The bounds for $\mathrm{H}$ were chosen based on experimental results. In the third step the analytical solution is fitted to the trial function over a frequency range from approximately $10^{-3}\left[\mathrm{~s}^{-1}\right]$ to approximately $3\left[\mathrm{~s}^{-1}\right]$, thereby $\mathrm{B}_{1}$ and $\mathrm{H}$ are held nearly constant and $\omega_{0}$ is varied over a small range to obtain the best fit of the phase slope.

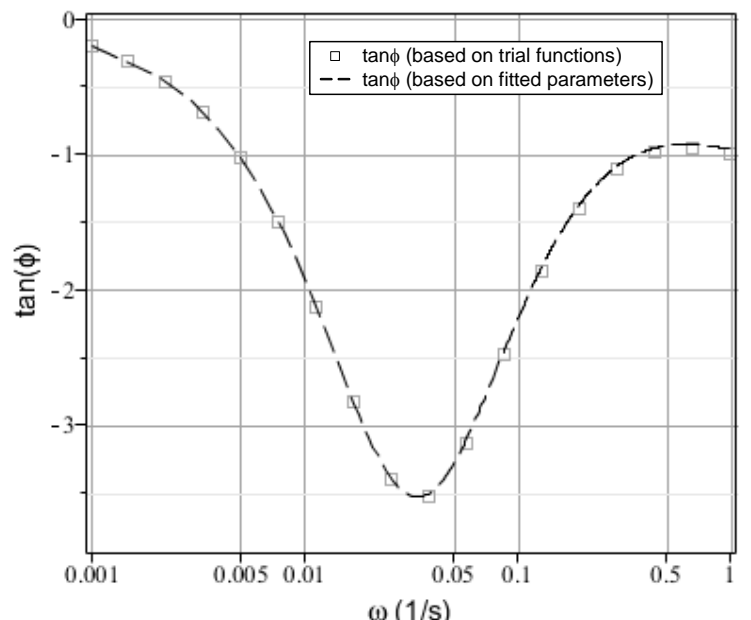

a) Phase slope based on trial function and the thereupon fitted phase slope

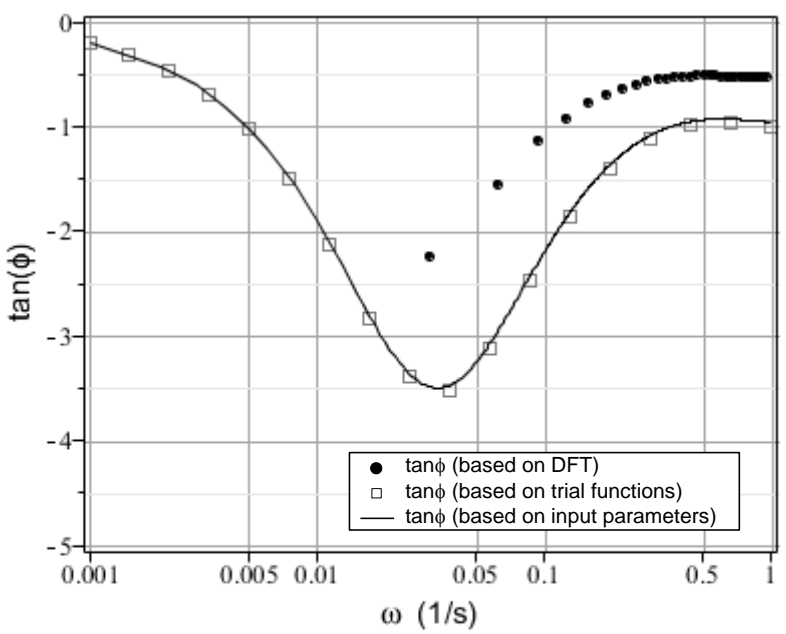

b) Comparison of the phase slope based on input parameters to the phase slopes of the trial function and the result of working with DFT

Fig. 5. Comparison of the phase slopes of the respective methods 
Figure 5a shows the fitted phase slope of the analytical solution of the heat conduction equation to the trial function of the simulated data over the relevant frequency range for the material parameter determining process. It can be seen that the fitting procedure in the PRFD procedure is highly suitable for fitting the phase slope of the analytical solution to the phase slope of simulated data. To show the capability of the trial function process in the time domain generally, the phase curve of the analytical solution with the included input parameters used in the simulations and the phase curve of the fitted trial function are shown in figure $5 \mathrm{~b}$. The diagram shows that the phase curve of the trial function coincides well with the phase curve of the analytical solution very well. Furthermore, the phase slope of the DFT applied to the simulated data is shown. The simulation parameters such as the sampling frequency and the simulation duration are chosen similar to measurements in practice. Therefore, a duration of $200 \mathrm{~s}$ and a sampling frequency of $50 \mathrm{~Hz}$ is used in the simulation. It can be seen, that it is not possible to obtain the correct phase curve with these simulation parameters using DFT.

\section{Numerical Simulation}

Numerical simulations based on FE-simulations were carried out with COMSOL Multiphysics to develop and optimise the PRFD procedure. The simulation model is based on a 1D solid of thickness $L$ and the material is assumed to be homogenous. The material parameters used in the simulations are similar to the characteristic of CFRP and are given in Table 1.

Table 1. Material parameters used in the FE-simulations.

\begin{tabular}{|l|l|l|l|}
\hline & symbol & value & unit \\
\hline thermal conductivity & $\mathrm{k}$ & 0.7 & $\mathrm{~W} \mathrm{~m} \mathrm{~K}^{-1}$ \\
\hline density & $\rho$ & 1490 & $\mathrm{~kg} \mathrm{~m}^{-3}$ \\
\hline specific heat capacity & $\mathrm{c}$ & 1200 & $\mathrm{Ws} \mathrm{kg}^{-1} \mathrm{~K}^{-1}$ \\
\hline
\end{tabular}

To simulate the time dependent temperature curve $T(0, t)$ as closely as possible to real measurement conditions, heat transfer coefficients of $h_{1}=13\left[\mathrm{~W} \mathrm{~m}^{-2} \mathrm{~K}^{-1}\right]$ and $\mathrm{h}_{2}=6\left[\mathrm{~W} \mathrm{~m}^{-2} \mathrm{~K}^{-1}\right]$ at the two boundaries $\mathrm{x}=0$ and $\mathrm{x}=\mathrm{L}$ are assumed. The boundary conditions at $x=0$ and $x=L$ are given in Eqs. (2) and (3).

The ambient temperature $T_{\infty}$ and the initial temperature $T_{0}$ are set at $293.15 \mathrm{~K}$. For the excitation term $q_{0} a$ pulse duration of $2 \mathrm{~ms}$ and a thermal source volumetric density of $1.5 \cdot 10^{6} \mathrm{Wm}^{-2}$ were chosen. In the FE-simulation model the temperature $T$ at $x=0$ has been simulated over a time range of $200 \mathrm{~s}$, similar to real PPT experiments in reflection mode.

\section{Measurement}

To evaluate the PRFD procedure measurement data were analysed. The measurements were carried out on a specimen, which exhibits different thicknesses. As the values of the FE-simulations were chosen to get approximate real test environments, the thicknesses of the analysed regions are the same as in the simulations and have the values 2.23 $\mathrm{mm}, 3.08 \mathrm{~mm}$ and $3.88 \mathrm{~mm}$.

The measurements are based on pulse thermography experiments in the reflection mode. To record the temperature profile an infrared camera (IRCam Equus $81 \mathrm{k} \mathrm{M}$ ) with a thermal sensitivity of $\leq 20 \mathrm{mK}$ in a spectral range of 3 to $5 \mu \mathrm{m}$ was used. Two flash lamps with a total electrical energy of $6 \mathrm{~kJ}$ and pulse duration of approximately 2 ms were used as a heat source. A measurement duration of $100 \mathrm{~s}$ and a frame rate of $50 \mathrm{~Hz}$ were chosen for the investigations.

\section{Results}

The suitability of the PRFD procedure to determine material parameter of pulse thermography data is being demonstrated on both FE-simulation data and on experimental measurement data.

\subsection{Numerical simulations}

All numerical simulations were realised as explained in paragraph 3 . The material parameters, shown in table 1 , are identical for all investigations. In the respective investigations only the length $L$ of the $1 D$ slab varies. The simulations were carried out for lengths of $2.23 \mathrm{~mm}, 3.08 \mathrm{~mm}$ and $3.88 \mathrm{~mm}$. Furthermore, the simulated data are superimposed with normally distributed noise to analyse the suitability of the PRFD procedure on noisy signals. 


\subsubsection{Numerical simulations without noise}

At first the PRFD procedure is tested on temperature profiles simulated by the FE-method without noise. As the material parameters which are used in the simulations are available, the accuracy of the PRFD procedure can be tested on this temperature data.

To depict the results of the PRFD procedure on simulated data, the simulated temperature profile and the fitted trial function for the thickness of $2.23 \mathrm{~mm}$ of the 1D slab are shown in figure $6 \mathrm{a}$. It can be seen that the physically correct trial functions enable a mathematical description of the simulated temperature profile. Excellent agreement between the trial function with the simulated temperature decay, as shown in figure $6 \mathrm{a}$, is necessary to obtain correct material parameters in the frequency domain.

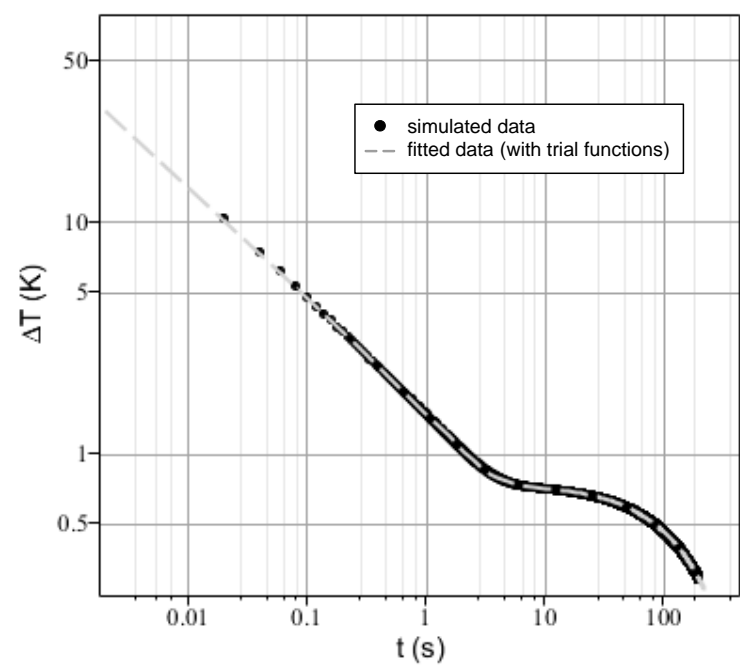

a) Temperature profile of simulated data and the temperature profile of the fitted trial function.

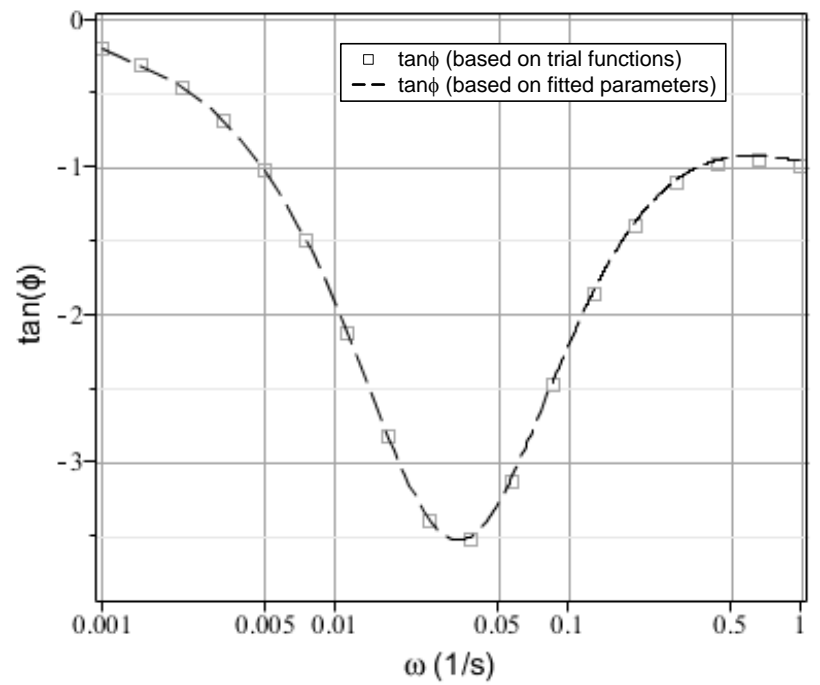

b) Phase curve of the trial function in comparison to the thereupon fitted phase slope.

Fig. 6. Results of the PRFD procedure for simulated data with a thickness of $L=2.23 \mathrm{~mm}$.

The phase curve of the trial function and the thereupon fitted phase curve are shown in figure $6 \mathrm{~b}$. It can be seen that the fitting procedure in the PRFD procedure enables an accurate fit of the analytical solution. The resulting material parameters of the PRFD procedure in comparison to the input parameters of the FE-simulations, for the various thicknesses are listed in table 2.

Table 2. Determined material parameters of the PRFD procedure compared to the input parameters of the FE-simulation

\begin{tabular}{|l|c|c|c|c|}
\hline \multicolumn{2}{|c|}{ Sample } & $\omega_{0}(1 / \mathrm{s})$ & $\mathrm{B} 1(-)$ & $\mathrm{H}(-)$ \\
\hline \multirow{3}{*}{ Simulation 2.23 mm } & parameters for simulation & $7,937 \mathrm{E}-02$ & $4,141 \mathrm{E}-02$ & 0,461 \\
\cline { 2 - 5 } & results obtained by PRFD & $8,074 \mathrm{E}-02$ & $3,990 \mathrm{E}-02$ & 0,480 \\
\cline { 2 - 5 } & relative deviation [\%] & 2,6 & $-3,6$ & 4,0 \\
\hline \multirow{3}{*}{ Simulation 3.04 mm } & parameters for simulation & $4,236 \mathrm{E}-02$ & $5,646 \mathrm{E}-02$ & 0,461 \\
\cline { 2 - 5 } & results obtained by PRFD & $4,310 \mathrm{E}-02$ & $5,483 \mathrm{E}-02$ & 0,480 \\
\cline { 2 - 5 } & relative deviation [\%] & 1,7 & $-2,9$ & 4,0 \\
\hline \multirow{3}{*}{ Simulation 3.88 mm } & parameters for simulation & $2,606 \mathrm{E}-02$ & $7,206 \mathrm{E}-02$ & 0,461 \\
\cline { 2 - 5 } & results obtained by PRFD & $2,644 \mathrm{E}-02$ & $7,109 \mathrm{E}-02$ & 0,458 \\
\cline { 2 - 5 } & relative deviation [\%] & 1,7 & $-1,3$ & $-0,7$ \\
\hline
\end{tabular}

In Table 2 it can be seen that the relative deviation of the determined material parameters 0obained by PRFD procedure compared to the parameters used in the simulation is less than 4\%. That shows the PRFD procedure is a suitable method to determine material parameters out of pulse thermography data. The results also show that the deviations of the determined parameter are reducing by increasing the length of the simulated specimen. 


\subsubsection{Numerical simulations with superimposed noise}

To investigate the stability of the PRFD procedure to determine material parameters of noisy data, the simulated temperature profiles described in the section before are superimposed by noise. The noise is set to be normal distributed and is limited at $\pm 40 \mathrm{mK}$. The simulated temperature profile and the fitted trial function for the thickness of $L=3.88 \mathrm{~mm}$ of the 1D slab are shown in Figure $7 \mathrm{a}$. It can be seen, that the fitting procedure of the PRFD procedure even enables a mathematical description of a noisy temperature curve. The superimposed noise has no significant influence to the fitted trial function. Figure $7 \mathrm{~b}$ shows the phase curve of the trial function and the thereupon fitted phase curve of the PRFD procedure.

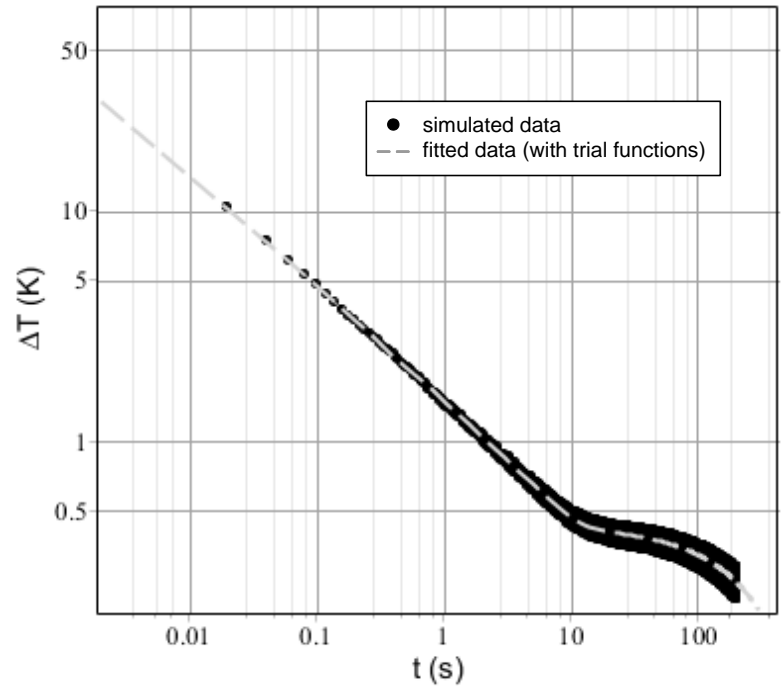

a) Temperature profile of simulated data with superimposed noise and the temperature profile of the fitted trial function.

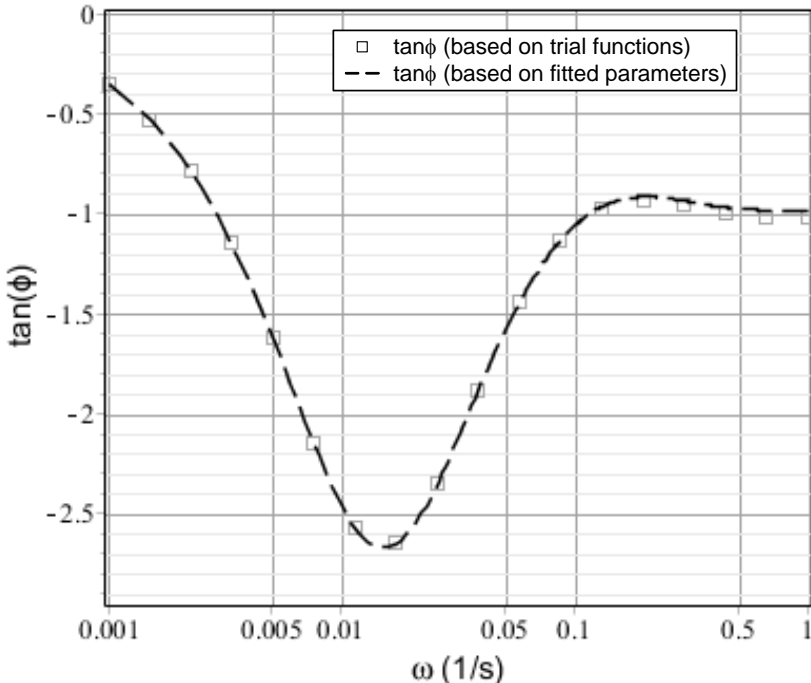

b) Phase curve of the trial function in comparison to the thereupon fitted phase slope.

Fig. 7. Results of the PRFD procedure for simulated data with superimposed noise. The simulation is performed with a thickness of $L=3.88 \mathrm{~mm}$.

The excellent agreement of the trial function with the simulated noisy data and the agreement of the phase slopes depict that the PRFD process is suitable to analyse noisy signals. The results of the PRFD procedure and the parameters used in the simulation are shown in table 3.

Table 3. Determined material parameters of the PRFD procedure compared to the input parameters of the FE-simulation by evaluating noisy signals.

\begin{tabular}{|l|l|c|c|c|}
\hline \multicolumn{2}{|c|}{ Sample } & $\omega_{0}(1 / \mathrm{s})$ & $\mathrm{B} 1(-)$ & $\mathrm{H}(-)$ \\
\hline \multirow{3}{*}{ Simulation $2.23 \mathrm{~mm}$ with noise } & parameters for simulations & $7,937 \mathrm{E}-02$ & $4,141 \mathrm{E}-02$ & 0,461 \\
\cline { 2 - 5 } & results obtained by PRFD & $8,080 \mathrm{E}-02$ & $3,990 \mathrm{E}-02$ & 0,480 \\
\cline { 2 - 5 } & relative deviation [\%] & 2,6 & $-3,7$ & 4,0 \\
\hline \multirow{3}{*}{ Simulation 3.04 mm with noise } & parameters for simulations & $4,236 \mathrm{E}-02$ & $5,646 \mathrm{E}-02$ & 0,461 \\
\cline { 2 - 5 } & results obtained by PRFD & $4,308 \mathrm{E}-02$ & $5,463 \mathrm{E}-02$ & 0,480 \\
\cline { 2 - 5 } & relative deviation [\%] & 1,7 & $-3,2$ & 4,0 \\
\hline \multirow{3}{*}{ Simulation 3.88 mm with noise } & parameters for simulations & $2,606 \mathrm{E}-02$ & $7,206 \mathrm{E}-02$ & 0,461 \\
\cline { 2 - 5 } & results obtained by PRFD & $2,663 \mathrm{E}-02$ & $7,102 \mathrm{E}-02$ & 0,453 \\
\cline { 2 - 5 } & relative deviation [\%] & 2,4 & $-1,4$ & $-1,8$ \\
\hline
\end{tabular}

The results of determined material parameters show that relative deviations to the simulation parameters are also less than 4\%. This implies that the PRFD procedure is a robust procedure for determining material parameters of noisy signals. 


\subsection{Measurements}

The suitability of the PRFD procedure for analysing real measurement data is investigated on three different areas of a sample which differ in the thickness $\mathrm{L}$. The recorded temperature profile and the fitted trial function for the measurement at the region of thickness $L=2.23 \mathrm{~mm}$ are depicted in figure $7 \mathrm{a}$. The result of the fitting procedure of the phase slope is shown in figure $7 \mathrm{~b}$.

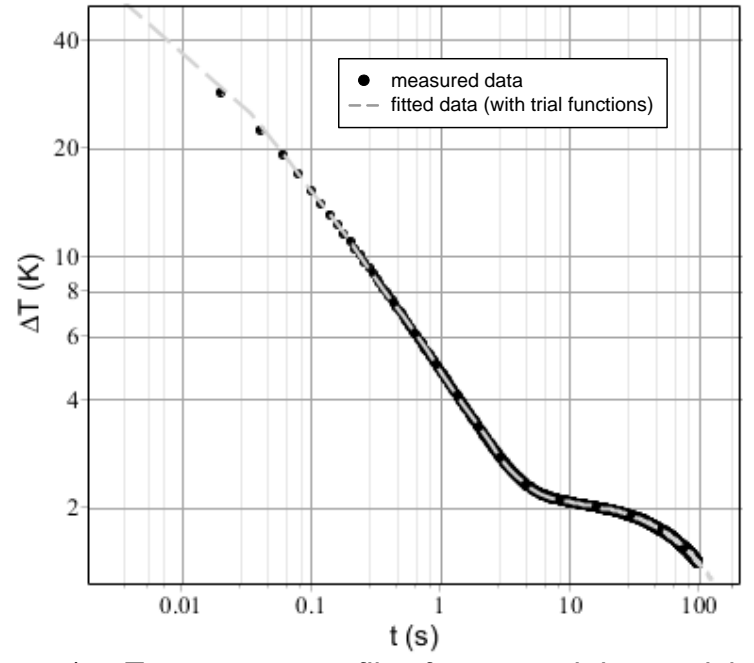

c) Temperature profile of measured data and the temperature profile of the fitted trial function.

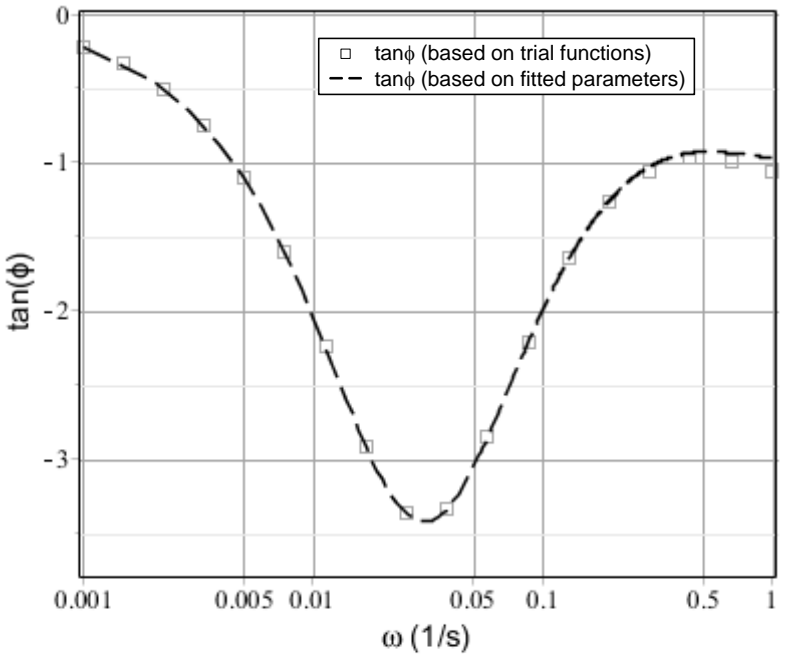

d) Phase curve of the trial function in comparison to the thereupon fitted phase slope.

Fig. 8. Results of the PRFD procedure for measured data with a thickness of $L=2.23 \mathrm{~mm}$.

The temperature profile of the trial function shows excellent agreement with the recorded temperature data. This shows that the trial function procedure is a suitable method for the description of the the temperature profile of recorded data mathematically.

The resulting phase profile of the trial function shows an excellent agreement with the thereupon fitted phase slope of the analytical solution. To validate the quantitative results of the PRFD process the results are compared to an alternative method. Thereby a time domain procedure which is closely related to the standardized Parker's method [6] is applied in the time domain to determine material parameters. This method is based on a transformation of the rear-face temperature in a semi-logarithmic domain $[7,8]$. The results of both methods are shown in table 4.

Table 4. Determined material parameters of the PRFD procedure compared to the determined parameters of the related Parker's method.

\begin{tabular}{|l|c|c|c|c|}
\hline \multicolumn{2}{|c|}{ Sample } & $\omega_{0}(1 / \mathrm{s})$ & $\mathrm{B} 1(-)$ & $\mathrm{H}(-)$ \\
\hline \multirow{4}{*}{ Measurement $2.23 \mathrm{~mm}$} & transmission method & $7,299 \mathrm{E}-02$ & $4,086 \mathrm{E}-02^{*}$ & $0,461^{*}$ \\
\cline { 2 - 5 } & results obtained by PRFD & $6,920 \mathrm{E}-02$ & $4,281 \mathrm{E}-02$ & 0,480 \\
\cline { 2 - 5 } & relative deviation [\%] & 5,2 & $-4,8$ & \\
\hline \multirow{3}{*}{ Measurement 3.08 mm } & transmission method & $4,112 \mathrm{E}-02$ & $5,646 \mathrm{E}-02^{*}$ & $0,461^{*}$ \\
\cline { 2 - 5 } & results obtained by PRFD & $3,942 \mathrm{E}-02$ & $5,670 \mathrm{E}-02$ & 0,480 \\
\cline { 2 - 5 } & relative deviation [\%] & 4,1 & $-0,4$ & \\
\hline \multirow{3}{*}{ Measurement 3.88 mm } & transmission method & $2,585 \mathrm{E}-02$ & $7,206 \mathrm{E}-02^{*}$ & $0,461^{*}$ \\
\cline { 2 - 5 } & results obtained by PRFD & $2,565 \mathrm{E}-02$ & $6,631 \mathrm{E}-02$ & 0,480 \\
\cline { 2 - 5 } & relative deviation [\%] & 0,8 & 8,0 & \\
\hline
\end{tabular}

* This parameters are determined by using approximation formulas.

The material parameters determined by the PRFD procedure are in a very good agreement with the parameters obtained by the alternative method. This indicates that the PRFD procedure is a suitable method for the quantitative evaluation of measurements. The advantage of the PRFD procedure is that three parameters can be determined without making assumptions, whereas Parker's likely method enables the determination of just one parameter. The parameters which are labelled with * were determined by using approximation formulas. 


\section{Conclusion}

This paper shows that the PRFD procedure enables the determination of three material parameters of pulse thermography data. The material parameters determined by the PRFD procedure show an excellent agreement to the parameters used in the FE-simulations. Furthermore the investigations show that noise, superimposed on the simulated temperature profile, does not have a significant influence on the determined material parameters. It can be said that the PRFD procedure is robust with regard to noisy signals. The investigations on real measurement data show a good agreement between the results of the PRFD procedure compared to the results of the Parker's likely method.

It can therefore be concluded that the PRFD procedure is a suitable method for the quantitative analysis of pulse thermography data.

In future works the PRFD procedure will be improved to avoid the currently required assumptions for the bounds of the parameter $\mathrm{H}$. The investigation of the stability of the PRFD procedure on further simulations and measurements will also be part of future works.

\section{Acknowledgements}

This work was financially supported by the TAKE OFF program of the Bundesministerium für Verkehr, Innovation und Technologie (BMVIT). Furthermore, we wish to thank our cooperation partner FACC AG.

\section{REFERENCES}

[1] Maldague X. and Moore P.O. "Theory and practice of Infrared Technology for nondestructive Testing", John Wiley \& Sons, 2001

[2] Stotter B., Gresslehner K.H., Mayr G., Hendorfer G. and Sekelja J. " Estimation of Material Parameters from Pulse Phase Thermography Data ", Proceedings of 40th Review of Progress in Quantitative Nondestructive Evaluation, paper QNDE2013-33 Baltimore, MD (USA), 2013.

[3] Marinetti S., Plotnikov Y., Winfree W.P., Braggiotti A. "Pulse phase thermography for defect detection and visualization" SPIE Conference Proceedings 3586, Newport Beach, CA, 1999, pp. 230-238

[4] Mandelis A. "Diffusion-Wave Fields - Mathematical Methods and Green Functions", Springer, 2001

[5] K.D. Cole et.al "Heat Conduction Using Green's Functions", John Wiley \& Sons, 2001

[6] Parker W. J., Jenkins, R. J., Butler, C. P. and Abbot, G. L., "Flash method of determining thermal diffusivity, heat capacity and thermal conductivity", Journal of Applied Physics, vol. 32, pp. 1679-1684, 1961.

[7] Thermitus M.-A. and Laurent M., "New logarithmic technique in the flash method". International Journal of Heat and Mass Transfer, vol. 40(17), pp. 4183-4190, 1997.

[8] Hendorfer G., Mayr G., Zauner G., Haslhofer M. and Pree R. "Quantitative determination of porosity by active thermography", AIP conference proceedings 894, pp. 702-708, Portland (Washington), 2006.

[9] D. P. Almond and P. M. Patel " Photothermal Science and Techniques", Chapman \& Hall,1996 